\title{
Antenatal corticosteroid use and clinical evolution of preterm newborn infants
}

\author{
Brazilian Neonatal Research Network*
}

\begin{abstract}
Objectives: To describe the use of antenatal corticosteroid and clinical evolution of preterm babies.

Methods: An observational prospective cohort study was carried out. All 463 pregnant women and their 514 newborn babies with gestational age ranging from 23 to 34 weeks, born at the Brazilian Neonatal Research Network units, were evaluated from August 1 to December 31,2001. The data were obtained through maternal interview, analysis of medical records, and follow-up of the newborn infants. Data analysis was performed with the use of chi-square, $t$ Student, MannWhitney, and ANOVA tests and multiple logistic regression, with level of significance set at $5 \%$.

Results: Treatment was directly associated with the number of prenatal visits, with maternal hypertension and with the antenatal use of tocolytic agents. Babies from treated pregnant women presented better Apgar scores at the 1st and 5 th minute, reduced need for intervention in the delivery room and lower SNAPPE II. They were born with higher birth weight, longer gestational age and needed less surfactant use, ventilation, and oxygenation time. After multiple logistic regression, the use of antenatal corticosteroid independently improved birth conditions, decreased ventilation time, being related to increased occurrence of neonatal sepsis.

Conclusions: The use of corticosteroid was associated with better prenatal care and birth conditions, better preterm evolution but higher risk of infection.
\end{abstract}

J Pediatr (Rio J). 2004;80(4):277-84: Antenatal glucocorticoids, prematurity, mechanical ventilation, infection, low birth weight.

\footnotetext{
* The following researchers participated in this study:

Hospital das Clínicas, Faculdade de Medicina de Ribeirão Preto, Universidade de São Paulo (USP), Ribeirão Preto, SP: Francisco E. Martinez, Nelson J. Linhares e Maria L. S. Ferlin.

Centro de Atenção Integral à Saúde da Mulher, Universidade Estadual de Campinas (UNICAMP), Campinas, SP: Sérgio Marba and Abimael A. Netto.

Hospital de Clínicas de Porto Alegre, Universidade Federal do Rio Grande do Sul (UFRGS), Porto Alegre, RS: Renato S. Procianoy and Natacha T. Uchoa.

Instituto Fernandes Figueira, Fundação Oswaldo Cruz, Rio de Janeiro, RJ: José Maria A. Lopes and Olga Bomfim.

Hospital São Paulo, Escola Paulista de Medicina, Universidade Federal de São Paulo (UNIFESP), São Paulo, SP: Ruth Guinsburg, Maria Fernanda B. Almeida, Milton Miyoshi and Joice F. Meneguel.

Berçário Anexo à Maternidade do Hospital das Clínicas de São Paulo, Universidade de São Paulo (USP), São Paulo, SP: Cléa R. Leone, Lílian S. R. Sadeck and Flávio A. C. Vaz.

Hospital São Lucas, Pontifícia Universidade do Rio Grande do Sul (PUCRS), Porto Alegre, RS: Renato M. Fiori, Humberto H. Fiori and Manuel R. Pereira.

Hospital das Clínicas, Faculdade de Medicina de Botucatu, Universidade do Estado de São Paulo (UNESP), Botucatu, SP: Cleide E. P. Trindade and Maria R. Betlin.

Financial support: This study was supported by the Ministry of Health.
}

Manuscript received Mar 10 2004, accepted for publication May 122004.

\section{Introduction}

In 1972, Liggins \& Howie showed that the use of antenatal corticosteroid therapy substantially reduces the incidence of respiratory distress syndrome and mortality in preterm infants. ${ }^{1}$ In a meta-analysis of 18 controlled studies carried out between 1972 and 1995, Crowley demonstrated that antenatal corticosteroid therapy reduced the incidence of respiratory distress syndrome by $47 \%$, decreased the mortality among preterm infants by $40 \%$, and reduced the incidence of intracranial hemorrhage by $52 \% .^{2}$

Despite the benefits obtained, the use of antenatal corticosteroid therapy does not seem to be a widespread therapeutic option. Thirteen international studies on the use of surfactant in preterm infants published between 1989 and 1994 clearly showed that corticosteroids were used irregularly. Their use in these studies ranged from 0.8 to $81 \% .^{3}$ More recently, a survey of 11,440 births in hospitals affiliated with the Canadian Neonatal Network showed that corticosteroids were used in $59 \%$ of preterm infants aged between 24 and 34 weeks. ${ }^{4}$ 
The scenario is quite similar in Brazil. For instance, at a teaching maternity hospital, 5 corticosteroids were administered to approximately $50 \%$ of patients who had premature births, or to $4 \%$ of those whose babies had been born before 34 weeks' gestation in seven maternity wards of public hospitals in Rio de Janeiro. ${ }^{6}$

The aim of the present study is to prospectively describe the frequency of use of antenatal corticosteroid therapy and the clinical outcome of infants born at or before 34 weeks' gestation in hospitals affiliated with the Brazilian Neonatal Network.

\section{Methods}

This prospective observational cohort study included all infants born after 23 weeks and before 35 weeks of gestation at hospitals and neonatal units affiliated with the Brazilian Neonatal Network between August 1 and December 31, 2001. The protocol study was approved by the local Research Ethics Committees.

Newborns with major congenital anomalies ${ }^{7}$ or chromosomal syndromes were excluded from the study. Newborns whose mothers had received antenatal corticosteroid therapy for any other reason than induction of fetal maturation (e.g.: to treat chronic diseases such as bronchial asthma and collagen diseases) were left out of the study as well.

A form was filled out for every eligible newborn while the mother was in hospital.

Maternal and neonatal variables were obtained from medical charts, prenatal card, and if possible, from prenatal care records, as well as from an interview with the mothers. All mothers signed an informed consent form before the beginning of the study.

The following maternal characteristics were considered: age, previous pregnancies, live births, number of prenatal appointments, diseases during current pregnancy, use of tocolytics and antenatal corticosteroids regardless of the number of doses, type of corticosteroid (betamethasone or dexamethasone) and length of administration before delivery.

The observed neonatal characteristics were the following: gestational age, appropriateness for gestational age, Apgar score at 1 and 5 minutes, procedures performed in the delivery room, SNAPPE II, neonatal outcome, length of hospital stay, and death.

Gestational age was expressed in weeks and was determined by obstetric estimation based on the date of last menstruation, ultrasound results or neonatal exams by the New Ballard ${ }^{8}$ score. The final gestational age was that which was calculated according to the date of last menstruation or to early fetal ultrasound when the difference between this date and the date determined by neonatal examination was less than one week. If the difference was greater than one week, the gestational age calculated by the New Ballard score was considered.

Appropriateness of intrauterine growth was classified according to the intrauterine growth curve developed by
Alexander et al. ${ }^{9}$ Newborns with birthweight between the 10 th and 90 th percentiles were considered to be appropriatefor-gestational age (AGA), those below the 10th percentile were regarded as small-for-gestational-age (SGA), and those above the 90 th percentile were considered large-forgestational-age (LGA).

In our setting, all neonatal units use the guidelines for neonatal resuscitation of the American Academy of Pediatrics, adopted by the Brazilian Society of Pediatrics. This allows determining the amount of bag-mask ventilation, tracheal intubation, cardiac massage, and use of medications. ${ }^{10}$ Apgar scores at 1 and 5 minutes were recorded for all infants. ${ }^{11}$

The Score for Neonatal Acute Physiology, Perinatal Extension, version II (SNAPPE-II) described by Richardson et al., ${ }^{12}$ was applied to all infants at 12 hours of life.

The following clinical outcomes and treatment requirements were observed during neonatal hospital stay:

- Respiratory distress syndrome: clinical and radiological diagnosis.

- Air leak syndrome: clinical and radiological diagnosis of pneumothorax, pneumomediastinum and/or interstitial emphysema.

- Use of exogenous surfactant.

- Necessity of conventional mechanical ventilation, continuous positive airway pressure (CPAP) or any other ventilation method.

- Necessity of oxygen therapy.

- Chronic pulmonary disease: necessity of oxygen therapy at 28 days of life and at 36 weeks of corrected age.

- Patent ductus arteriosus (PDA), treated with indomethacin and/or surgical closure.

- Diagnosis of sepsis: determined by positive blood culture.

- Antibiotic therapy for over 5 days.

- Necrotizing enterocolitis: age at clinical, radiological or surgical diagnosis, classification from I to III. ${ }^{13}$ All grades were taken into consideration.

- Intraventricular hemorrhage (IVH), classified according to Papile et al. ${ }^{14}$ Grades I to IV were determined by transfontanellar ultrasound performed between the fifth and fourteenth days of life or after some clinical instability that could cause or increase the hemorrhage. Grades III and IV were registered.

- In-hospital mortality: age in hours or days calculated according to birth date.

The SPSS 10.0 for Windows was used for the statistical analysis. The chi-square test or Fisher's exact test, Student's $t$ test and the Mann-Whitney test, ANOVA and multiple logistic regression were used. A significance level of $5 \%$ was adopted for all tests.

Multiple logistic regression was used to determine the variables more strongly associated with perinatal conditions, necessity of mechanical ventilation, occurrence of infection and death. The Apgar score at 1 minute lower than 3 or greater than or equal to 3 was used as dependent variable 
for perinatal conditions. In the logistic regression used to assess the necessity of mechanical ventilation, the use or not of a mechanical ventilator during the hospital stay was adopted as the dependent variable. To assess the occurrence of infection, we used the presence or absence of positive blood culture as dependent variable. The dependent variable used to assess mortality was death and no death.

\section{Results}

During the study period, 463 women gave birth to 514 preterm babies with 34 weeks of gestation or less. The main characteristics of mothers, who received or not at least one dose of antenatal corticosteroid, are shown in Table 1.

The patients were young, $58(12.5 \%)$ were aged 18 years or less. Most of them were inexperienced, since 68 (14.7\%) were pregnant for the first time, 191 (41.3\%) had their first live birth, and 109 (23.6\%) did not have any prenatal appointment.

Some characteristics of the patients who received corticosteroid therapy were significantly different from those who did not receive this medication. Treated patients had had a larger number of previous pregnancies, more prenatal appointments, a more frequent diagnosis of hypertension, and higher use of tocolytics.

Some general characteristics of the 514 infants, classified according to the use or not of antenatal corticosteroid therapy by their mothers, are shown in Table 2 .

We observed that infants whose mothers received antenatal corticosteroid therapy had some different characteristics from those whose mothers had not received this medication. At birth, these infants were more mature (regarding both age and weight), were predominantly female and born by cesarean section, comparatively to those who had not been submitted to antenatal corticosteroid therapy.

Table 3 shows perinatal conditions, necessity of medical care in the delivery room and SNAPPE II of infants whose mothers had or had not received antenatal corticosteroid therapy.

Infants whose mothers had received corticosteroids had significantly better Apgar scores at 1 and 5 minutes, with less necessity for interventions in the delivery room. Infants whose mothers had not been treated with corticosteroid therapy showed a worse health status at 12 hours of life, as indicated by SNAPPE II.

We used the Apgar score at 1 minute, lower than 3 or greater than or equal to 3 , and logistic regression to determine which variables were associated with perinatal conditions. First we defined which variables could be individually associated with the Apgar score at 1 minute. The variables number of prenatal appointments, maternal hypertension, use of corticosteroids, use of tocolytics, type of delivery, gestational age, birthweight, and gender were significantly associated and were then included in the logistic regression model. Of these variables, antenatal use of at least one dose of corticosteroid, gestational age, and being small for gestational age remained statistically significant. These three variables showed a protective effect, that is, they increased the chances of the Apgar score at 1 minute being greater than 3 . The protective effect of these variables is shown in Table 4.

Table 1 - The main characteristics of mothers who received or not at least one dose of antenatal corticosteroid

\begin{tabular}{lcccc}
\hline & All mothers & Corticosteroid & No corticosteroid & p* \\
\hline Number & 463 & $284(61.3 \%)$ & $179(38.7 \%)$ & \\
Age (years) & $26.8 \pm 7.1$ & $27.4 \pm 7.3$ & $25.9 \pm 6.7$ & 0.06 \\
Previous pregnancies & $2.48 \pm 2.21$ & $2.66 \pm 2.31$ & $2.21 \pm 2.02$ & 0.03 \\
Alive children & $1.25 \pm 1.62$ & $1.26 \pm 1.63$ & $1.22 \pm 1.62$ & 0.82 \\
Prenatal medical visits & $3.53 \pm 3.1$ & $4.27 \pm 3.1$ & $2.41 \pm 2.5$ & $<0.01$ \\
Maternal diabetes & $18(3.9 \%)$ & $14(3.0 \%)$ & $4(0.9 \%)$ & 0.14 \\
Maternal infection & $120(26 \%)$ & $77(16.6 \%)$ & $43(9.3 \%)$ & 0.54 \\
Premature rupture & & & & \\
of membranes for $>$ & $93(20.1 \%)$ & $57(12.3 \%)$ & $36(7.7 \%)$ & 0.89 \\
18 hours & & & & \\
Hypertension & $146(31.6 \%)$ & $108(23.3 \%)$ & $38(8.2 \%)$ & $<0.01$ \\
Use of tocolytics & $87(18.8 \%)$ & $79(17.1 \%)$ & $8(1.7 \%)$ & $<0.01$ \\
\hline
\end{tabular}

* Comparison between the groups that used or not antenatal corticosteroid. 
Table 2 - Characteristics of infants, classified according to the use or not of antenatal corticosteroid therapy by their mothers

\begin{tabular}{lcccc}
\hline & All & Corticosteroid & No corticosteroid & p* \\
\hline Number of infants & 514 & $315(61.3 \%)$ & $199(38.7 \%)$ & \\
Weight $(\mathrm{g})$ & $1,309 \pm 486$ & $1,353 \pm 443$ & $1,241 \pm 543$ & 0.01 \\
Gestational age (weeks) & $30.3 \pm 2.8$ & $30.8 \pm 2.2$ & $29.5 \pm 3.5$ & $<0.01$ \\
Small for gestational age & $138(26.8 \%)$ & $86(27.3 \%)$ & $52(26.1 \%)$ & 0.77 \\
Female & $247(48.1 \%)$ & $164(52.1 \%)$ & $83(41.7 \%)$ & 0.03 \\
Cesarean delivery & $323(62.8 \%)$ & $214(67.9 \%)$ & $109(54.8 \%)$ & $<0.01$ \\
\hline
\end{tabular}

* Comparison between mothers who used or not corticosteroid.

Some information about the clinical outcome of the assessed infants is described in Table 5 . For the sake of analysis, the infants were classified based on the fact whether their mothers had or had not received at least one dose of antenatal corticosteroid therapy.

The diagnosis of hyaline membrane disease was comparable in both groups, but infants whose mothers had received antenatal corticosteroid therapy needed less exogenous surfactant, less mechanical ventilation, and less oxygen therapy. On the other hand, the total length of oxygen therapy and necessity of long-term oxygen therapy was similar in both groups.
The incidence of intracranial hemorrhage, grades III and IV, and the necessity of drug or surgical treatment of the patent ductus arteriosus was the same in both groups.

With regard to the incidence of infection, infants whose mothers had received antenatal corticosteroid therapy had a larger number of positive blood cultures, had to use a larger amount of antibiotics, and a higher percentage of infants developed necrotizing enterocolitis of any type, independently of how many cycles of corticosteroids they had received. However, these infants had a better survival rate.

Table 3 - Perinatal conditions, necessity of medical care in the delivery room of infants whose mothers had or had not received antenatal corticosteroid therapy

\begin{tabular}{lcccc}
\hline & $\begin{array}{c}\text { All } \\
(\mathbf{n}=\mathbf{5 1 4})\end{array}$ & $\begin{array}{c}\text { Corticosteroid } \\
(\mathbf{n}=\mathbf{3 1 5})\end{array}$ & $\begin{array}{c}\text { No corticosteroid } \\
(\mathbf{n = 1 9 9 )}\end{array}$ & p* \\
\hline Apgar at 1 minute $<3$ & $143(27.8 \%)$ & $61(19.4 \%)$ & $82(41.2 \%)$ & $<0.01$ \\
Apgar at 5 minutes $\geq 7$ & $429(83.5 \%)$ & $288(91.4 \%)$ & $141(70.9 \%)$ & $<0.01$ \\
Necessity of: & & & & \\
$\quad$ Bag-mask ventilation & $227(44.2 \%)$ & $136(43.2 \%)$ & $91(45.7 \%)$ & 0.57 \\
$\quad$ Intubation & $160(31.1 \%)$ & $71(22.5 \%)$ & $89(44.7 \%)$ & $<0.01$ \\
$\quad$ Cardiac massage & $37(7.2 \%)$ & $10(3.2 \%)$ & $27(13.6 \%)$ & $<0.01$ \\
$\quad$ Medications & $29(5.6 \%)$ & $5(1.6 \%)$ & $24(12.1 \%)$ & $<0.01$ \\
SNAPPE II & $12.1 \pm 18.7$ & $10.5 \pm 14.9$ & $14.7 \pm 23.4$ & 0.01 \\
\hline
\end{tabular}

* Comparison between mothers who used or not corticosteroid. 
Table 4 - Multiple logistic regression of the variables associated with values of Apgar at 1 minute (Apgar $<3=0$; Apgar $\geq 3=1$ )

\begin{tabular}{lccc}
\hline Variable & Odds ratio & C.I.* (95\%) & p \\
\hline Antenatal use & & & \\
of corticosteroid & 2.47 & $1.43-4.30$ & $<0.01$ \\
Gestational age & 1.38 & $1.19-1.60$ & $<0.01$ \\
Small for & 1.97 & $1.06-3.60$ & 0.03 \\
gestational age & & &
\end{tabular}

* Confidence interval.

Logistic regression was used to assess the influence of multiple variables associated with the necessity of mechanical ventilation, presence of positive blood culture, and survival rate.

The fact whether the infants were or were not submitted to mechanical ventilation was used as dependent variable, while those individually associated with this variable were considered independent variables. The following variables were included in the model: number of prenatal appointments, antenatal use of tocolytics (yes or no), antenatal use of corticosteroid (yes or no), gestational age, Apgar score at 1 minute and SNAPPE II. Table 6 shows those variables that were not excluded from the model after logistic regression.
After logistic regression, antenatal use of corticosteroids, gestational age and Apgar score at 1 minute were considered protective variables regarding the necessity of mechanical ventilation. SNAPPE II was considered a risk variable. The remaining variables were excluded during logistic regression since they did not show statistical significance.

The fact whether the infants had or did not have at least one positive blood culture while in hospital was defined as dependent variable. Those individually associated with blood culture were considered independent variables. The following variables were included in the model: maternal infection (yes or no), antenatal use of corticosteroids (yes or no), premature rupture of membranes for over 18 hours (yes or no), gestational age (in weeks), birthweight (in grams), Apgar score at 1 minute and SNAPPE II, respiratory distress syndrome (yes or no), necessity of mechanical ventilation (yes or no), pneumothorax (yes or no), patent ductus arteriosus (yes or no), necrotizing enterocolitis (yes or no), intraventricular hemorrhage grades III or IV (yes or no) and necessity of oxygen therapy after 36 weeks' corrected age (yes or no). Table 7 shows the variables that were not excluded after logistic regression.

Birthweight was the only variable with a protective effect that was not excluded. The variables antenatal use of corticosteroids, mechanical ventilation, and oxygen after 36 weeks' corrected age were related to an increased risk of positive blood cultures.

Variables individually and significantly associated with death were considered independent variables in the logistic

Table 5 - Clinical outcome of the assessed infants whose mothers had or had not received at least one dose of antenatal corticosteroid therapy

\begin{tabular}{lccc}
\hline & $\begin{array}{c}\text { Antenatal use } \\
\text { of corticosteroid } \\
(\mathbf{n}=\mathbf{3 1 5})\end{array}$ & $\begin{array}{c}\text { No } \\
\text { corticosteroid } \\
\mathbf{( n = 1 9 9 )}\end{array}$ & p \\
\hline Hyaline membrane & $147(46.7 \%)$ & $101(50.8 \%)$ & 0.37 \\
Use of surfactant & $85(27 \%)$ & $87(43.7 \%)$ & $<0.01$ \\
Mechanical ventilation & $130(41.3 \%)$ & $114(57.3 \%)$ & $<0.01$ \\
Days with mechanical ventilation & $4.52 \pm 11.7$ & $6.57 \pm 15.9$ & 0.09 \\
Air leak & $16(5.1 \%)$ & $15(7.5 \%)$ & 0.25 \\
Need of oxygen & $218(69.2 \%)$ & $156(78.4 \%)$ & 0.02 \\
Days with oxygen & $11.6 \pm 21.4$ & $14.1 \pm 29.3$ & 0.25 \\
Oxygen at 28 days & $53(16.8 \%)$ & $35(17.6 \%)$ & 0.82 \\
Oxygen at 36 weeks & $33(10.5 \%)$ & $24(12.1 \%)$ & 0.57 \\
Intraventricular hemorrhage grades III or IV & $23(7.3 \%)$ & $19(9.5 \%)$ & 0.41 \\
Need of treatment of ductus arteriosus & $33(10.5 \%)$ & $28(14.1 \%)$ & 0.14 \\
Positive blood culture & $60(19 \%)$ & $21(10.6 \%)$ & $<0.01$ \\
Use of antibiotics & $194(61.6 \%)$ & $96(48.2 \%)$ & $<0.01$ \\
Necrotizing enterocolitis (all) & $21(6.7 \%)$ & $5(2.5 \%)$ & 0.03 \\
Necrotizing enterocolitis (Bell's grade $>1)$ & $13(4.1 \%)$ & $3(1.5 \%)$ & 0.12 \\
Survival rate & $272(86.3 \%)$ & $135(67.8 \%)$ & $<0.01$ \\
\hline
\end{tabular}


Table 6 - Variables that were not excluded from the model after logistic regression regarding the need of mechanical ventilation of newborns

\begin{tabular}{lccc}
\hline Variable & Odds ratio & C.I.* (95\%) & p \\
\hline $\begin{array}{l}\text { Antenatal use of } \\
\text { corticosteroid }\end{array}$ & 0.51 & $0.29-0.89$ & 0.01 \\
Gestational age & 0.60 & $0.53-0.69$ & $<0.01$ \\
Apgar at 1 minute & 0.85 & $0.77-0.93$ & $<0.01$ \\
SNAPPE II & 1.09 & $1.06-1.12$ & $<0.01$
\end{tabular}

* Confidence interval.

regression. The following variables were included in the model: number of prenatal appointments, antenatal use of tocolytics (yes or no), antenatal use of corticosteroids (yes or no), maternal hypertension (yes or no), gestational age (in weeks), Apgar score at 1 minute, SNAPPE II, necessity of mechanical ventilation (yes or no), pneumothorax (yes or no) and intraventricular hemorrhage grades III or IV (yes or no). Table 8 shows the variables that were not excluded from the model after logistic regression.

Table 7 - Variables that were not excluded after logistic regression regarding the presence of positive blood culture in the newborns

\begin{tabular}{lccc}
\hline Variable & Odds ratio & C.I.* (95\%) & p \\
\hline $\begin{array}{l}\text { Antenatal use of } \\
\text { corticosteroid }\end{array}$ & 2.87 & $1.52-5.39$ & $<0.01$ \\
$\begin{array}{l}\text { Birth weight } \\
\text { Mechanical ventilation }\end{array}$ & 0.99 & $0.99-1.00$ & 0.02 \\
Oxygen at 36 weeks & 2.23 & $1.05-4.73$ & 0.04 \\
\hline
\end{tabular}

* Confidence interval.

Table 8 - Variables that were not excluded from the model after logistic regression regarding newborns' death

\begin{tabular}{lccc}
\hline Variable & Odds ratio & C.I.* (95\%) & p \\
\hline $\begin{array}{l}\text { Antenatal use of } \\
\text { tocolytics }\end{array}$ & 0.23 & $0.08-0.61$ & $<0.01$ \\
Maternal hypertension & 0.43 & $0.20-0.86$ & 0.02 \\
Gestational age & 0.75 & $0.65-0.86$ & $<0.01$ \\
SNAPPE II & 1.02 & $1.01-1.04$ & 0.02 \\
Mechanical ventilation & 6.62 & $2.35-18.6$ & $<0.01$ \\
\hline
\end{tabular}

* Confidence interval.
Antenatal use of tocolytics, maternal hypertension and gestational age were protective variables in relation to death. SNAPPE II and necessity of mechanical ventilation were risk factors for death.

\section{Discussion}

A controlled double-blind clinical trial is the best method to evaluate the efficiency of a drug. In the present study, we assessed the effects of the antenatal use of corticosteroids using a prospective observational cohort study, which is certainly a methodological limitation. However, given the current knowledge about the antenatal use of corticosteroids, ${ }^{2}$ using a different study design would be inappropriate.

It should be noted that over $60 \%$ of the assessed mothers had received antenatal corticosteroids. The current knowledge about the efficiency of this therapy recommends that all mothers at risk of premature birth should be treated, ${ }^{2}$ but this is not always possible. ${ }^{15}$ Even in industrialized countries, the use of antenatal corticosteroid therapy is not so common as it should be. ${ }^{4}$ Interestingly enough, the characteristics of mothers who received antenatal corticosteroids were different from those who did not. We may say that mothers who received antenatal corticosteroids were more experienced and had a better prenatal care. However, maternal characteristics and the clinical outcome of infants were not different when mothers received complete or incomplete cycles of antenatal corticosteroids. Therefore, these mothers and their infants were assigned to the same study group.

The better prenatal care in women who had received antenatal corticosteroids is of utmost importance, since this shows that the more contact with the patient, the better the possibilities of preventive management. Interestingly enough, the fact that some mothers were hypertensive did not prevent them from receiving the drug. After the use of antenatal corticosteroids was regarded as a way to improve the pulmonary function of preterm infants, it has been suggested that maternal hypertension could be a contraindication to the therapy. ${ }^{1}$ This issue remains controversial until today, 15 but it did not impose any restriction on the treatment of mothers. Quite on the contrary, hypertension was associated with a larger number of treatments. This suggests that the closer follow-up provided to the patients may allay the fear of using corticosteroids in hypertension, increasing the supply of corticosteroids to these patients. The association between the use of tocolytics and corticosteroids supports the hypothesis that these mothers had a better medical care. During preterm labor, tocolytics may be used to reverse the process or allow time for the corticosteroid to take effect. ${ }^{2}$

As occurred with maternal characteristics, the characteristics of infants whose mothers received antenatal corticosteroids were different from untreated ones. The infants were older and weighed more and were born by cesarean section. The longer intrauterine life of these 
infants and the higher incidence of C-sections probably result from the better prenatal care these mothers received instead of from the direct effect of the drug.

By analyzing the perinatal conditions of infants, we obtained interesting information. By and large, infants with less than 34 weeks of gestational age constitute a high-risk group. Nearly half of them required bag-mask ventilation in the delivery room and approximately one third required tracheal intubation. In other words, these infants should be given birth to in a delivery room especially prepared for adequate ventilatory support. This type of support was efficient in all evaluated services, where more than $80 \%$ of the newborns had their ventilatory parameters normalized within five minutes.

The group of newborns exposed to antenatal corticosteroid therapy showed better conditions at birth. When intervention in the delivery room was necessary, infants whose mothers had received corticosteroids showed better conditions. These infants needed fewer aggressive interventions in the delivery room, such as tracheal intubation, cardiac massage or use of epinephrine. It should be underscored that the protective effect of antenatal corticosteroid therapy can still be detected at 12 hours of life using SNAPPE II. The protective effect of the corticosteroid as well as that of gestational age and of intrauterine growth retardation on perinatal conditions persisted after the logistic regression. The better perinatal conditions obtained with antenatal corticosteroid therapy have not been but should be widely advertised. ${ }^{16}$

The first benefit of the antenatal use of corticosteroids was the improvement of pulmonary function. ${ }^{1}$ By analyzing variables individually, we may say that the infants whose mothers received antenatal corticosteroids had a better respiratory performance. Differently from the results reported in the literature, ${ }^{17}$ the diagnosis of hyaline membrane disease was comparable in both groups. However, infants whose mothers received antenatal corticosteroids required less exogenous surfactant, less mechanical ventilation and less oxygen therapy and needed fewer days on artificial ventilation. On the other hand, the total length of oxygen therapy and the necessity of long-term oxygen supply were similar in both groups. After logistic regression, the antenatal use of corticosteroids, gestational age and Apgar score at 1 minute were protective factors against mechanical ventilation. SNAPPE II, however, was a risk factor, that is, the higher its value, the higher the necessity of mechanical ventilation. This was obviously expected, since SNAPPE II is a severity score. ${ }^{12}$

The antenatal use of corticosteroids is described as a protective factor against intraventricular hemorrhage ${ }^{2}$ and as a possible risk factor for neonatal infection. ${ }^{18}$ The protective effect against intraventricular hemorrhage could not be demonstrated in the patients assessed in the present study.

Infants whose mothers were treated with antenatal corticosteroids had a higher incidence of positive blood cultures, more frequent use of antibiotics and a higher percentage of them developed necrotizing enterocolitis of any type. As infant characteristics were quite different, logistic regression was used to determine which factors were actually associated with the risk of infection, described as presence of positive blood culture. After the analysis, antenatal use of corticosteroids, birthweight, mechanical ventilation, and necessity of oxygen at 36 weeks of corrected age were statistically significant. Of these variables, only birthweight had a protective effect; the remaining variables were considered risk factors, as expected. It should be highlighted that mechanical ventilation and oxygen at 36 weeks are associated with late infection, which is related to neonatal intensive care. The evidence provided in the literature on antenatal corticosteroid therapy and risk of infection is contradictory. Whereas the meta-analysis carried out by Crowley ${ }^{2}$ does not indicate high risk, other authors suggest that the risk of infection is increased, 4,19 as shown in the present study, too. As the literature mentions that the benefits of corticosteroid therapy decrease after 34 weeks of gestation, it has been suggested that the therapy should be used only in infants older than 34 weeks whose lung immaturity has been confirmed. 20

Certainly, the better survival rate is the most important outcome in the present study. Survival rate is a parameter that somewhat summarizes the success of the whole set of measures with which risk patients are provided. Infants whose mothers received antenatal corticosteroids had a better survival. Logistic regression plays a crucial role in the analysis of factors associated with survival.

Notably, perinatal factors were strongly associated with survival. The antenatal use of tocolytics, maternal hypertension and advanced gestational age are factors that increase the chances of survival, and upon which pediatricians can have little influence. The antenatal use of corticosteroids was not statistically significant. Anyway, prenatal care is fundamental for the prognosis.

SNAPPE II and mechanical ventilation were associated with higher risk of death. SNAPPE II indicates infant conditions within the first 12 hours of life, ${ }^{12}$ and as the other parameters, it also indicates the strong influence of perinatal conditions on the prognosis.

The necessity of mechanical ventilation had a huge effect on clinical outcome. Despite the improvements made to new respirators and to mechanical ventilation procedures, our data still show that the necessity of mechanical ventilation is a high risk for survival. It should be recalled that it is the most severely ill infants who need ventilatory support.

The present study assessed a cohort of infants treated at teaching hospitals affiliated with the Brazilian Neonatal Network and can be used for comparison with other Brazilian health services. Mothers who received antenatal corticosteroids showed characteristics that indicate better prenatal care, and consequently their infants had a better birthweight and were born at a more advanced gestational age. The antenatal use of corticosteroids was directly associated with better perinatal conditions and higher risk of infection. 


\section{References}

1. Liggins GC, Howie RN. A controlled trial of antepartum glucocorticoid treatment for prevention of the respiratory distress syndrome in premature infants. Pediatrics. 1972;50:515-25.

2. Crowley P. Prophylactic corticosteroids for preterm birth. Cochrane Database Syst Rev. 2002;(3):CD000065.

3. Ryan CA, Finer NN. Antenatal corticosteroid therapy to prevent respiratory distress syndrome. J Pediatr. 1995;126:317-9.

4. Chien L, Ohlsson A, Seshia MMK, Boulton J, Sankaran K, Lee SK. Variations in antenatal corticosteroid therapy: a persistent problem despite 30 years of evidence. Obstet Gynecol. 2002;99:401-8.

5. Albuquerque ICC, Amorim MMR, Meneses J, Katz L, Santos LC. Avaliação do impacto da corticiterapia antenatal para a aceleração da maturidade pulmonar fetal nos recém-nascidos em maternidade escola brasileira. RBGO. 2002;24:655-61.

6. Krauss-Silva L, Costa TP, Reis AF, Iamada NO, Azevedo AP, Albuquerque CP. Avaliação da qualidade da assistência hospitalar obstétrica: uso de corticóides no trabalho de parto prematuro. Cad Saúde Pública. 1999;15:817-29.

7. Chung CS, Myrianthopoulos NC. Congenital anomalies: mortality and morbidity, burden and classification. Am J Med Genet. 1987;27:505-23.

8. Ballard JL, Khoury JC, Wedig K, Wang L, Eilers-Walsman BL, Lipp R. New Ballard Score, expanded to include extremely premature infants. J Pediatr. 1991;119:417-23.

9. Alexander GR, Himes JH, Kaufman RB, Mor J, Kogan M. A United States National Reference for fetal growth. Obstet Gynecol. 1996;87:163-8.

10. Niermeyer S, Kattwinkel J, Van Reempts P, Nadkarni V, Phillips B, Zideman D, et al. International Guidelines for Neonatal Resuscitation: An excerpt from the Guidelines 2000 for Cardiopulmonary Resuscitation and Emergency Cardiovascular Care: International Consensus on Science. Contributors and Reviewers for the Neonatal Resuscitation Guidelines. Pediatrics. 2000;106(3):E29.

11. Apgar V. A proposal for a new method of evaluation of the newborn infant. Curr Res Anesth Analg. 1953;32:260-7.

12. Richardson DK, Corcoran JD, Escobar GJ, Lee SK. SNAP-II and SNAPPE-II: Simplified newborn illness severity and mortality risk scores J Pediatr. 2001;138:92-100.
13. Walsh MC, Kliegman RM. Necrotizing enterocolitis: treatment based on staging criteria. Pediatr Clin North Am. 1986;33: 179-201.

14. Papile LA, Burstein J, Burstein R, Koffler $H$. Incidence and evolution of subependymal and intraventricular hemorrhage: a study of infants with birth weights less than 1,500 gm. J Pediatr. 1978;92:529-34.

15. Bloom SL, Leveno KJ. Corticosteroid use in special circumstances: preterm ruptured membranes, hypertension, fetal growth restriction, multiple fetuses. Clin Obstet Gynecol. 2003;45: 150-60.

16. Gardner MO, Goldenberg RL, Gaudier FL, Dubard MB, Nelson KG, Hauth JC. Predicting low Apgar scores of infants weighing less than 1000 grams: the effect of corticosteroids. Obstet Gynecol. 1995;85:170-4.

17. Banks BA, Macones G, Cnaan A, Merrill JD, Ballard JD, Ballard RA, et al. Multiple courses of antenatal corticosteroids are associated with early severe lung disease in preterm neonates. J Perinatol. 2002;22:101-7.

18. Vermillion ST, Soper DE, Chasedunn-Roak J. Neonatal sepsis after betamethasone administration to patients with preterm premature rupture of membranes. Am J Obstet Gynecol. 1999;181:320-7.

19. Gunkel JH, Mitchell BR. Observational evidence for the efficacy of antenatal steroids from randomized studies of surfactant replacement. Am J Obstet Gynecol. 1995;173:281-5.

20. ACOG Committee Opinion. Antenatal corticosteroid therapy for fetal maturation. Obstet Gynecol. 2002;99:871-3.

Corresponding author:

Francisco Eulógio Martinez

Departamento de Puericultura e Pediatria

Faculdade de Medicina de Ribeirão Preto - USP

Av. Bandeirantes, 3900

CEP 14049-900 - Ribeirão Preto, SP, Brazil

Tel.: (16) 602.2808 - Fax: (16) 602.2700

E-mail: femartin@fmrp.usp.br 